\title{
Differential regulation of NPR-B/GC-B by protein kinase $C$ and
} calcium

\author{
Sarah E Abbey-Hosch, Dmitri Smirnov and Lincoln R Potter*
}

\author{
Address: Department of Biochemistry, Molecular Biology and Biophysics, University of Minnesota, Minneapolis, MN, 55455, USA \\ Email: Lincoln R Potter* - potter@umn.edu \\ ${ }^{*}$ Corresponding author
}

from 2nd International Conference of cGMP Generators, Effectors and Therapeutic Implications

Potsdam, Germany, 10-12 June, 2005

Published: 16 June 2005

BMC Pharmacology 2005, 5(Suppl I):PI doi:I0.1 I86/I47I-22I0-5-SI-PI

C-type natriuretic peptide (CNP) activation of the guanylyl cyclase-linked natriuretic peptide receptor-B (NPR-B) stimulates vasorelaxation and bone growth. Hormones and phorbol esters (PMA) inhibit NPR-B in calcium- and protein kinase $\mathrm{C}$-dependent manners, respectively. Here, we characterize the kinetic properties of NPR-B in membranes from cells exposed to PMA, the calcium ionophore, ionomycin, or sphingosine-1-phosphate (S1P). PMA and ionomycin primarily increased the $K_{m}$ and decreased the $\mathrm{V}_{\max }$ of NPR-B for GTP, respectively, whereas S1P caused modest changes in both parameters. PMA and S1P treatment increased the $\mathrm{EC}_{50}$ for CNP activation by 8 and 3-fold, whereas ionomycin was ineffective. All three agents caused NPR-B dephosphorylation, but the basis for the loss of phosphate differed between treatments. In vitro phosphorylation of NPR-B in membranes was markedly diminished by prior PMA or S1P exposure, whereas ionomycin pretreatment had no effect. The involvement of the known phosphorylated residues in each process was tested with a mutant receptor containing glutamates substituted for these sites. While the effect of PMA was lost on this receptor, the effects of S1P and ionomycin were only partially blocked. Our data suggest that the molecular bases for PMA- and calcium-dependent inhibition of NPR-B are unique. The former results from reduced phosphorylation of a known site and primarily affects the affinity of NPR-B for CNP and GTP. The latter is associated reductions in maximal velocities by a mechanism that does not involve inhibition of NPR-B phosphorylation and requires a process in addition to the dephosphorylation of the known sites. 